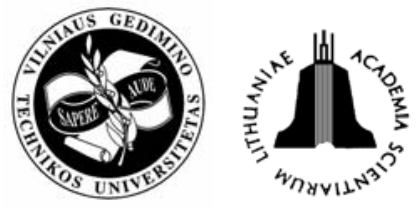

\title{
PROBABILITY MODELS FOR ASSESSING TRANSPORT TERMINAL OPERATION
}

\author{
Adolfas Baublys \\ Transport Research Institute, Vilnius Gediminas Technical University, \\ Plytinès g. 27, LT-10105 Vilnius, Lithuania.E-mail: baublys@takas.lt
}

Received 1 September 2006; accepted 4 December 2006

\begin{abstract}
The paper considers the following problems: 1) The development of a probability model to determine malfunction of the terminal; 2) Determining the emergency situations at the terminal based on statistical data; 3) Optimizing the effect of failures on the operation of the terminal; 4) Identifying the conflicting situations in making managerial decisions at the terminal.
\end{abstract}

Keywords: transport terminal; malfunction of the terminal; probability models.

\section{Introduction}

Terminals play an important role in freight transportation.

It can be observed that various random factors influence strictly regulated terminal operation. If these factors are not taken into account by deterministic models in planning and controlling terminal operation, its efficiency can be considerably decreased.

The analysis of the well-known journals dealing with transport problems "TRANSPORTATION SCIENCE”, "TRANSPORT THEORY AND STATISTICAL PHYSICS”, "TRANSPORTATION RESEARCH PART $B$ - METHODOLOGICAL" published in the last 20 years has shown that statistical-stochastic models are suggested to be used in this area [1-4]. However, the articles considering the role of random factors as well as their formation and classification could not be found. These factors can emerge for subjective and objective reasons.

Factors caused by objective reasons may be divided into three groups including technical, organizational and economic aspects. The main technical factors are associated with the process of transportation and equipment (e.g. vehicles, containers, hoisting mechanisms, etc.) and with the technological aspects; organizational factors include specialization, scientific and technical information, measures aimed at increasing quality of transport services, work of the customs, moral responsibility for the quality of work, etc., while economic factors are related to tariffs, work payment, labour intensity, financial incentives, etc.

Generally, technical-economic indicators of the terminal operation should be analyzed in terms of random factors which are random with respect to any argument value as well. The arguments include time or other parameters of the terminal operation (technological process). Thus, the criteria of optimality should also be considered as being random rather than determined.

Most criteria used to assess the operation of the terminal and individual technological processes are interlinked and this should be taken into account when using them as optimality criteria. In determining the numerical characteristics of technical-economic indicators according to statistical data obtained in the process of terminal operation, mathematical expectation as well as correlation and variance functions should be calculated.

\section{A probability model for determining interferences in terminal operation}

The state of the terminal when after a certain accident it cannot operate normally will be referred to as a failure. In the reliability theory a failure (discontinuity of operation) is an event leading to the situation when the parameters of a system do not meet the specified limits. In our case, a failure is considered to be a case when the terminal cannot normally perform its functions.

The reliability criterion is referred to as a probability to avoid an inadmissible limiting state in the terminal operation when trying to reach the level defined by some planned indicator $R_{i}$, actually implying failurefree terminal operation.

If the planned indicator $i$ which is a function of random parameters $r_{1}, \ldots, r_{k}$ is denoted by $R_{i}\left(r_{1}, \ldots, r_{k}\right)$, while $Q_{i}\left(q_{i}, \ldots, q_{k}\right)$ and this indicator are also a function of random parameters $\left(q_{1}, \ldots, q_{l}\right)$ at the considered moment, then, the mode of operation not reaching the limiting state will be expressed as follows:

$$
R_{i}\left(r_{1}, \ldots, r_{k}\right)-Q_{i}\left(q_{i}, \ldots, q_{k}\right)=x_{i}>0
$$


To assess the state of the terminal at a particular moment, total distribution density of the values $x_{1}, x_{2}, x_{3}, \ldots, x_{i}$, i.e. $\omega\left(x_{1}, x_{2}, x_{3}, \ldots, x_{i}\right)$ should be known. If the function is known, then probability of non-occurrence of a limiting state with respect to all indicators may be described by the equation:

$$
\begin{aligned}
& P\left[x_{1}>0, x_{2}>0, x_{3}>0, \ldots, x_{i}>0\right]= \\
& \int_{0}^{\infty} \int_{0}^{\infty} \int_{0}^{\infty} \ldots \int_{0}^{\infty} \omega\left(x_{1}, x_{2}, x_{3}, \ldots, x_{i}\right) d x_{1}, \mathrm{~d} x_{2}, \mathrm{~d} x_{3}, \ldots, \mathrm{d} x_{i} .
\end{aligned}
$$

In general, if all values $x_{1}, x_{2}, x_{3}, \ldots, x_{i}$ are assumed to be correlated as well as having normal distribution, then the formula given below may be used to express the function $\omega\left(x_{1}, x_{2}, x_{3}, \ldots, x_{i}\right)$ :

$$
\begin{aligned}
& \frac{\omega_{i}\left(x_{1}, \ldots, x_{i}\right)=}{\sigma_{1 x}, \ldots, \sigma_{n x} \sqrt{(2 \pi)^{2} D}} \times \\
& \exp \left(-\frac{1}{2 D} \sum_{i=1}^{n} \sum_{j=1}^{m} D_{i j} \frac{x_{i}-a_{i}}{\sigma_{i} x_{i}} \times \frac{x_{j}-a_{j}}{\sigma_{j} x_{j}}\right),
\end{aligned}
$$

where $D$ is a determinant of the row $n ; D_{i j}$ is algebraic complement of the determinant $D$ of the element $r_{i j} ; r_{i j}$ is a coefficient describing a static relationship between random values $x_{1}, \ldots, x_{i}$.

A determinant of the row $n$ is as follows:

$$
D=\left|\begin{array}{cccc}
1 & r_{12} & \ldots & r_{1 n} \\
r_{21} & 1 & \ldots & r_{2 n} \\
\ldots & \ldots & \ldots & \ldots \\
r_{n 1} & . & \ldots & 1
\end{array}\right|, \quad r_{i j}=r_{j i}, \quad\left|r_{i j}\right| \leq 1 .
$$

In practice, all indicators of terminal performance do not reach their limiting values. Usually, one or two indicators have to be evaluated. In the case of two indicators distributed according to the normal function $\omega\left(x_{1}, x_{2}\right)$, the formula used for two-dimensional distribution function of two random values $(n=2)$ may be applied:

$$
\begin{aligned}
& \left(r_{12}=r_{21}=r\right), \\
& D=\left|\begin{array}{ll}
1 & r \\
r & 1
\end{array}\right|, \\
& D_{11}=D_{21}=1 \\
& D_{12}=D_{21}=2, \\
& \varpi_{2}\left(x_{1}, x_{2}\right)=\frac{1}{2 \pi \sigma_{1} \sigma_{2} \sqrt{1-r^{2}}} \times \\
& \exp \left\{-\frac{1}{2\left(1-r^{2}\right)}\left[\frac{\left(x_{1}-a_{1}\right)^{2}}{\sigma_{1}^{2}}-2 r \frac{\left(x_{1}-a_{1}\right)\left(x_{2}-a_{2}\right)}{\sigma_{1} \sigma_{2}}+\right.\right. \\
& \left.\left.\frac{\left(x_{2}-a_{2}\right)^{2}}{\sigma_{2}^{2}}\right]\right\},
\end{aligned}
$$

where $a_{1}$ and $a_{2}$ are average values of $x_{1}$ and $x_{2}$, and $\sigma_{1}^{2}$ and $\sigma_{2}^{2}$ mean square deviation of the values of $x_{1}$ and $x_{2}$.

The probability $P\left[x_{1}>0 ; x_{2}>0\right]$ is calculated by the formula:

$$
P\left[x_{1}>0 ; x_{2}>0\right]=\int_{0}^{\infty} \int_{0}^{\infty} \omega\left(x_{1} x_{2}\right) d x_{1} d x_{2} .
$$

Substituting the value $\omega\left(x_{1}, x_{2}\right)$ into the formula (1) and rearranging it respectively, we will obtain:

$$
\begin{aligned}
& P\left[x_{1}>0 ; x_{2}>0\right]=\frac{1}{2} F\left(\alpha_{1}\right)+\frac{1}{2} F\left(\alpha_{2}\right)- \\
& T\left(\alpha_{1}, \bar{\alpha}_{1}\right)-T\left(\alpha_{2}, \bar{\alpha}_{2}\right)+\frac{1}{2}
\end{aligned}
$$

where

$$
\begin{aligned}
& \alpha_{1}=\frac{a_{1}}{\sigma_{1}} ; \quad \alpha_{2}=\frac{a_{2}}{\sigma_{2}} ; \\
& \bar{a}_{1}=\frac{\alpha_{2}-r \alpha_{1}}{\alpha_{1} \sqrt{1-r^{2}}} ; \\
& \bar{a}_{2}=\frac{\alpha_{2}-r \alpha_{2}}{\alpha_{2} \sqrt{1-r^{2}}} ; \\
& F\left(\alpha_{1}\right)=\frac{1}{\sqrt{2 \pi}} \int^{\alpha_{1}} \mathrm{e}^{-y^{2} / 2} d y \text { the Gauss function; }
\end{aligned}
$$
$T\left(\alpha_{1}, \bar{\alpha}_{1}\right)$ is obtained from the tables.

A simplified formula for $P\left[x_{1}>0 ; x_{2}>0\right]$ may be obtained when $x_{1}$ and $x_{2}$ are not in correlation, i.e. when $r=0$ :

$$
P\left[x_{1}>0 ; x_{2}>0\right]-0.25\left[1+F\left(\alpha_{1}\right)\right]\left[1+F\left(\alpha_{2}\right)\right] .
$$

Failures at the terminal are usually caused by some emergency situations. Therefore, in considering the latter, it could be advisable to use theoretical methods based on the probability value functions.

\section{Determining emergency situations at the terminal based on statistical information}

Let us assume that no emergencies occur at the terminal for some time, then they happen for the time $\tau_{1}^{\prime \prime}$ and later, in the time period $\tau_{2}^{\prime}$, the terminal is operating normally. Then, during the time period $\tau_{3}^{\prime}$ terminal operation is influenced by another random factor, and so on.

Let us refer to the moments

$$
t_{n}^{\prime}=\sum^{n=1}\left(\tau_{j}^{\prime}+\tau_{j}^{\prime \prime}\right), \quad n=1,2, \ldots, \tau_{0}^{\prime}=\tau_{0}^{\prime \prime}=0,
$$

corresponding to the end of the period $\tau_{n}^{\prime}-1$ as a failure.

The moments

$$
t_{n}^{\prime \prime}=\sum_{j=0}^{n=1}\left(\tau_{j}^{\prime}+\tau_{j}^{\prime \prime}\right)+\tau_{n}^{\prime}, \quad n=1,2, \ldots, \tau_{0}^{\prime}=\tau_{0}^{\prime \prime}=0,
$$


corresponding to the end of the period $\tau_{n}^{\prime}$ will be referred to as recovery.

If all values $\tau_{n}^{\prime}$ and $\tau_{n}^{\prime \prime}$ are assumed to be independent, all $\tau_{n}^{\prime}$ are uniformly distributed according to the law $F(t)=P\left\{\tau_{n}^{\prime} \leq t\right\}$ with a mathematical expectation $T_{1}=M\left[\tau_{n}^{\prime}\right]$ and the variance $\sigma_{1}^{2}=D\left[\tau_{n}^{\prime}\right]$. All periods $\tau_{n}^{\prime \prime}$ are uniformly distributed according to the law $G(t)=P\left\{\tau_{n}^{\prime \prime} \leq t\right\} \quad$ with mathematical expectation $T_{2}=M\left[\tau_{n}^{\prime \prime}\right]$, and the laws $F(t)$ and $G(t)$ have continuous densities $f(t)=F^{\prime}(t)$ and $q(t)=G^{\prime}(t), \quad \sigma_{1}^{2}+\sigma_{0}^{2} \neq 0$, respectively (for not taking into account a typical case). Then, the considered process is an ordinary recovery process with the finite time of recovery. The average number of failures in terminal operation in the time period $(0, t)$ is referred to as the recovery function,

$$
H(t)=\sum_{n=1}^{\infty} G^{(n)}(t),
$$

where $G^{(n)}(t)-n$-multiple convolution $G(t)$.

The rate of failures at the terminal is expressed as:

$$
h(t)=H^{\prime}(t)=q(t)+\int_{0}^{t} h(t+z) q(z) d z,
$$

where $z$ is integration variable.

The analysis of the statistical data has shown that the flows of individual failures are ordinary, allowing us to assume that $h(t) \equiv h, h_{i}(t) \equiv h_{h}$, where $i$ is the index of the type of failure.

The periods of various operational states are distributed according to the exponential law:

$$
g(t)=1-\mathrm{e}^{h t} .
$$

When the recovery periods are of the uniform Poisson nature, the distribution of the periods is as follows:

$$
F(t)=\sum_{i=1}^{m} \frac{h_{i}}{h} F_{i}(t),
$$

where $m$ is the number of various types of failures.

The average time of failure-free operation can be obtained from the equation (2):

$$
T_{2}=\int_{0}^{\infty} \mathrm{e}^{-h t}=\frac{1}{h},
$$

while the average time of recovery is:

$$
T_{1}=\int_{0}^{\infty}[1-F(t)] d t=\sum_{i=1}^{m} \frac{h_{i}}{h} T_{1 i} .
$$

The stability coefficient is as follows:

$$
K_{s}=\frac{1}{1+\sum_{i=1}^{m} \frac{T_{1 i}}{T_{2 i}}} .
$$

The stability of terminal operation is expressed in the following way:

$$
P(t)=K_{s} \exp \left(-\frac{t}{T^{2}}\right) .
$$

Let us refer to the sum of all recovery periods $\tau_{n}^{\prime}$ as the total input in the recovery time until the moment $t$ and denote it as $N_{t}$. The analysis has shown that when $t \rightarrow \infty$, the value of $N_{t}$ approaches normal distribution with mathematical expectation expressed as:

$$
M\left[N_{t}\right] \approx \frac{T_{1}}{T_{1}+T_{2}} t
$$

and the variance

$$
D\left[N_{t}\right] \approx \frac{T_{1}^{2} T_{2}^{2}\left(\frac{\sigma_{1}^{2}}{T_{1}^{2}}+\frac{\sigma_{2}^{2}}{T_{2}^{2}}\right)}{\left(T_{1}+T_{2}\right)^{3}} t .
$$

The data on the characteristics of the flow of failures at the terminal are given in Table.

Major characteristics of the flows of failures at the terminal

\begin{tabular}{|l|c|c|c|c|}
\hline \multicolumn{1}{|c|}{ Characteristic } & \multicolumn{3}{c|}{ Cause (type) of failure } \\
\cline { 2 - 5 } & $\begin{array}{c}\text { No freight } \\
\text { for carriage }\end{array}$ & Crane failure & Truck failure & $\begin{array}{c}\text { Lack of service } \\
\text { personnel }\end{array}$ \\
\hline Average input to failure $B_{i}=M\left[\delta_{k}(i)\right.$, in hours & 89,4 & 45,6 & 142,6 & 126,4 \\
\hline Failure rate $\lambda_{i}=1 / B_{i}$, failures/hour & 0,017 & 0,025 & 0,014 & 0,008 \\
\hline $\begin{array}{l}\text { Average number of failures in a 5-day week with two- } \\
\text { shift work } v_{i}(t)\end{array}$ & 2,9 & 75,3 & 1,75 & 1,63 \\
\hline $\begin{array}{l}\text { Probability of occurrence of empirical distribution } \\
\text { values } \sigma_{n}(i) \text { with theoretical } P\left\{H^{2}\right\} \text { according to } \\
\text { pearson }\end{array}$ & 0,99 & 0,978 & 0,95 & 0,956 \\
\hline $\begin{array}{l}\text { Reliability function of management system } \\
W_{i}(t)=P\left\{\delta_{n}(i)>t\right\}\end{array}$ & $\mathrm{e}^{-0,017 t}$ & $\mathrm{e}^{-0,025 t}$ & $\mathrm{e}^{-0,014 t}$ & $\mathrm{e}^{-0,008 t}$ \\
\hline $\begin{array}{l}\text { Distribution of failures per week of operation } \\
P_{i}^{(n)}(t)=P\left\{v_{i}(t)\right\} \leq n\end{array}$ & $\frac{2,9^{n}}{n !} \mathrm{e}^{-2,9}$ & $\frac{2,3^{n}}{n !} \mathrm{e}^{-2,3}$ & $\frac{1,75}{n !} \mathrm{e}^{-1,75}$ & $\frac{1,63^{n}}{n !} \mathrm{e}^{-1,63}$ \\
\hline
\end{tabular}




\section{Optimization of failure influence on the terminal operation}

A great number of factors influence the operation of the terminal and may cause its malfunction. However, their influence varies to a great extent. Therefore, they are divided into major and minor factors. In this case, the simulation results should be optimized before using them for management purposes.

The problem may be formulated as follows. Let the controlled variable be a random number with the domain function $F(x)$. The range (domain) of the random number $x$ (interval $[A B]$ ) is subdivided into $N$ non-intersecting steps by the points $x_{0}=A<x_{1}<x_{2} \ldots<x_{N-1}<x_{N}=B$. As mentioned above, a number of minor random factors $y$ play a role alongside the main controlled variable $x$. Thus, the random number $x$ in $z=x+y$ should be assessed.

Let us denote the distribution function of the random number $y$ by $f_{x}(y)$. For managerial (planning) purposes, let us take the average value of the function $f(i, j)$, where $f(i, j)$ is a penalty for deciding to prescribe the controlled variable to step $j$, while the value $x$ is actually found in step $i$.

Minor factors may cause the situation when the controlled variable with its value $z_{1}, z_{2}, \ldots, z_{N-1}$ not matching the limits of steps $x_{1}, x_{2}, \ldots, x_{N-1}$ appears to be the best.

The optimization problem may be formulated in the following way: to find the vector $\left\{z_{i}\right\}_{i=1}^{N-1}$ minimizing

$$
M_{x}(i, j)=\sum_{i=1}^{N} \sum_{j=1}^{N} P(i, j) f(i, f),
$$

where $P_{i, j}$ is probability of the event when $x$ is found in the step $i-m$, while $(x+y)$ is in the $j-t h$ step.

The problem will be solved by the dynamic programming method. In this case, this method is advantageous because no special conditions concerning $F(x)$ and $f_{x}(y)$ are specified. The following recurrent relationships will be used to determine optimal values of the controlled variable. First, let us calculate the value functions:

$$
S_{k}(\alpha, \beta)=\sum_{i=1}^{N} P\left\{x \in\left[x_{i-1}, x_{i}\right] z \in[\alpha, \beta]\right\} f(i, k),
$$

where $S_{k}(\alpha, \beta)$ is an average penalty corresponding to the case when the value $z$ is in the interval $[\alpha, \beta]$ under condition that the interval is in step $k$. In this case, $\alpha$ and $\beta$ range from $A^{*}$ to $B^{*}, A^{*}=\min \left\{x+y_{x}\right\}$, $B^{*}=\max \left\{x+y_{x}\right\}$, and it can easily be observed that:

$$
P\left\{x \in\left[x_{i-1}, x_{i}\right], z \in[\alpha, \beta]\right\}=d F(x)^{\beta-x} \int_{\alpha-x}^{-x} d f_{x}(y) .
$$

The functions given below can be found at other parts of the recurrent relationship:

$$
T\left(\left[\alpha, B^{*}\right], m\right)=\min \sum_{j=N-m+1}^{N} \sum_{i=1}^{N} P(i, j) f(i, j),
$$

where the minimum is obtained by dividing the interval $\left\lfloor\alpha, B^{*}\right\rfloor$ into $m$ sections by points:

$$
\begin{aligned}
& z_{N-m}^{(m)}\left(\alpha-B^{*}\right)= \\
& \alpha<z_{N-m+1}^{(m)}\left(\alpha, B^{*}\right)<\ldots<z_{N}^{(m)}\left(\alpha, B^{*}\right)=B^{*} .
\end{aligned}
$$

According to Bellman's optimality principle:

$$
\begin{aligned}
& T\left(\alpha, B^{*}, m\right)= \\
& \min _{(m)}\left\{S_{N-m+1}\left(\alpha, z_{N-m+1}^{(m)}\right)+T\left[\left(z_{N-m+1}^{(m)}, B^{*}\right) m-1\right]\right\}
\end{aligned}
$$

The expressions (3) and (4) comprehensively describe the recurrent relationship. Beginning with the second step, it is possible to calculate

$$
\begin{aligned}
& \left.T\left(\mid \alpha, B^{*}\right\rfloor, m\right) \text { and } z_{i}^{(m)}\left(\alpha, B^{*}\right), \\
& l=N-m+1, \ldots, N-1,
\end{aligned}
$$

while in the step $N-m$ we find

$$
T([A, B], N)=\min _{\left\{z_{j}\right\}_{j=1}^{N}} m f(i, j),
$$

which is actually the solution of the problem.

The above algorithm can be expanded by slightly modifying it to include the case when the penalty for inaccurate solution depends on the exact value of the controlled variable.

\section{Conflicting situations in decision-making}

In making optimal statistical decisions, the average number of conflicting situations should be determined. Conflicting situations occur due to the conflict of interests of the terminal authorities and carriers as well as of individual carriers and the terminal referring to a given and the future moment, etc.

Causes of the conflicting situations make a finite set $J$ and are associated with the stochastic nature of the terminal. Let us number the causes of the conflicting situations as follows: $i_{\alpha}=J, \alpha=1,2, \ldots, m$.

Conflicting situations should be divided into primary and secondary. The conflicting situations $i \in J$ are referred to as primary. They occur due to one cause which is usually associated with some emergency situation at the terminal.

Let us consider the determination of a random number of conflicting situations in time $(0, t)$.

The flow of events $(m+1)$ is given: flows of events $F_{1}, F_{2}, \ldots, F_{m}$ cause conflicting situations $i \in J, \alpha=1,2, \ldots, m$, while the flow $F_{m+1}$ indicates the superposition of the flows.

The moments of conflict $i$ occurrence $\Theta_{n}(i)$ are distributed on time axis with intervals $\delta_{n}(i)$ so that 
$\Theta_{n}(i)=\sum_{j=0}^{n-1} \delta_{i}(i), n=1,2, \ldots ; \quad i=1,2, \ldots, m ; \quad \delta_{0}(i)=0$,

while the moments of the event occurrence in the total flow are distributed at random intervals $\delta_{n}$ so that $\Theta_{n}=\sum_{j=0}^{n-1} \delta_{j}, n=1,2, \ldots, \delta_{0}=0$. Let us state that: a) all values $\delta_{n}(i)$ and $\delta_{n}$ are independent; b) all periods $\delta_{n}(i)$ are uniformly distributed according to the laws $W_{i}(t)=P\left\{\delta_{n}(i)>t\right\}$ with mathematical expectation $B_{i}=M\left[\delta_{n}(i)\right]$ and the variance $\sigma_{i}^{2}=D\left[\delta_{n}(i)\right]$; c) all periods $\delta_{n}$ are uniformly distributed according to the laws $W(t)=P\left\{\delta_{n}>t\right\}$ with mathematical expectation $B=M\left[\delta_{n}\right]$ and the variance $\sigma^{2}=D\left[\delta_{n}\right]$; d) the laws $W_{i}(t)$ and $W(t)$ have continuous densities $\omega_{i}(t)=-W_{i^{\prime}}(t) ; \omega(t)=W^{i}(t)$, respectively. A random variable $v_{i}(t)$ equal to the number of conflicting situations of the type $i$ at the moment $t$ may be found from the equation $v_{i}(t)<t<v_{i}(t)+1$. The value $v_{i}(t)$ may be only a positive integer. Let us note that

$$
\begin{aligned}
& P\left\{v_{i}(t) \geq n\right\}=P\left\{\Theta_{n}(i)<t\right\}= \\
& P\left\{\delta_{1}(i)+\delta_{2}(i)+\ldots+\delta_{n-1}(i)-t\right\}=W_{i}^{(n)}(t),
\end{aligned}
$$

where $W_{i}^{(n)}(t)$ is $v_{i}(t)=1, \ldots, v_{i}(t)=n$ of the sum of the distribution laws and uniformly distributed values expressed by a circuital integral

$$
W_{i}^{(n)}(t)=\int_{0}^{t} W_{i}^{(n-1)}(t-\delta) d W(\delta), W_{1}^{(1)}(t)=W(t) .
$$

It follows from (5) that

$$
P_{i}^{(n)}(t)=P\left\{v_{i}(t)=n\right\}=W_{i}^{(n)}(t)-W_{i}^{(n+1)}(t) .
$$

The average number of the $i$-type conflicting situations taking place prior to the moment $t$ is as follows:

$$
\begin{aligned}
& v_{i}(t)=M\left[v_{i}(t)\right]=\sum_{n=1}^{\infty} n P_{i}^{(n)}(t)= \\
& \sum_{n=1}^{\infty} n\left[W^{(n)}(t)-W_{i}^{(n+1)}(t)\right]= \\
& \sum_{n=1}^{\infty} n W_{i}^{(n)}(t)-\sum_{n=2}^{\infty}(n-1) W_{i}^{(n)}(t)=\sum_{n=1}^{\infty} W_{i}^{(n)}(t) .
\end{aligned}
$$

The average number of all kinds of conflicting situations in time $(0, t)$ may be expressed as:

$$
\mathrm{v}(t)=M[\mathrm{v}(t)]=\sum_{i=1}^{m} M\left[\mathrm{v}_{i}(t)\right]=\sum_{i=1}^{m} \mathrm{v}_{i}(t)-\sum_{i=2}^{m} \sum_{n=1}^{\infty} W_{i}^{(n)}(t) .
$$

The average number of conflicting situations per unit of time (if $t \rightarrow 0$ ) indicates a breakdown threat to the control system of the terminal, and

$$
v_{i}(t)=v_{i}^{\prime}(t), \quad v(t)=v^{\prime}(t)=\sum_{i=1}^{m} v_{i}(t) .
$$

It follows from (6) that breakdown threat may be expressed as:

$$
\mathrm{v}_{i}(t)=\sum_{n=1}^{\infty} \omega_{i}^{(n)}(t), \quad v(t)=\sum_{i=1}^{m} \omega^{(n)}(t)
$$

where $\omega_{i}^{(n)}(t)=\frac{d W_{i}^{(n)}(t)}{d t}, \omega^{(n)}(t)=\frac{d W^{(n)}(t)}{d t}$.

The periods when managerial, forwarding, technological and carriage operations are actually stable and the flows $F_{1}, F_{2}, \ldots, F_{m}$ satisfy the conditions of maintaining stationary and ordinary states and the influence condition, therefore, they are simple or ordinary Poisson processes. Since ordinary flows are independent of each other and any of them is a Poisson flow, therefore, the total flow is also a Poisson flow with the parameter equal to the sum of the constituent parameters. Then,

$$
\begin{aligned}
& \omega_{i}(t)=\lambda_{i} \mathrm{e}^{-\lambda_{i} t}, \quad \lambda_{i}=\frac{1}{B_{i}}, \\
& \omega(t)=\lambda_{i} \mathrm{e}^{-\lambda t}, \quad \lambda=\frac{1}{B}=\sum_{i=1}^{m} \frac{1}{B_{i}}
\end{aligned}
$$

of the reliability function

$$
W_{i}(t)=1-\mathrm{e}^{-\lambda_{i} t}, W(t)=1-\mathrm{e}^{-\lambda t} .
$$

The $i$-type conflicting situations have the following distribution:

$$
P_{i}^{(n)}(t)=P\left\{\mathrm{v}_{i}(t)=n\right\}=\frac{(\lambda t)^{n 1}}{n !} \mathrm{e}^{-\lambda_{i} t},
$$

where $\lambda_{i} t=v_{i}(t)$, while $v(t)$ is distributed according to the law:

$$
P^{(n)}(t)=P\{\mathrm{v}(t)=n\}=\frac{(\lambda t)^{n}}{n !} \mathrm{e}^{-\lambda t},
$$

where $\lambda t=v(t)$; while $P^{(0)}=1-W(t)=\mathrm{e}^{-\lambda t}$.

Breakdown threats are $v_{i}(t)=\lambda_{i}, v(t)=\lambda=\sum_{i=1}^{m} \lambda_{i}$.

If we want to determine the number of conflicting situations over a long period of time, then it is assumed that a random variable $v(t)$ is an asymptotic normal value with an average $M[\mathrm{v}(t)] \approx t / B$ and the variance $D[v(t)] \cong \sigma^{2} t / B^{3}$. Then, the number of conflicting situations over the time $(0, t)$ has the probability $1-\varepsilon$ and is in the range of

$t / B-U_{\varepsilon / 2^{\sigma}} \sqrt{t} / B^{3 / 2}<v_{i}(t)<t / B+U_{\varepsilon / 2^{\sigma}} \sqrt{t} / B^{3 / 2}$,

where $U_{\varepsilon / 2}$ is a quantile of the normal distribution with the probability $1-\varepsilon$ obtained from the table of normal distribution according to the condition: 


$$
\frac{1}{\sqrt{2 \pi}} \int_{-U_{\varepsilon / 2}}^{+U_{\varepsilon} / 2} e_{2}^{-x^{2}} d x=1-\alpha ; \quad x=\frac{v_{i}(t)-M\left[v_{i}(t)\right]}{\sqrt{D\left[v_{i}(t)\right]}} .
$$

The maximum amount of conflicting situations expected with the probability $1-\alpha$ over the time $(0, t)$ will be as follows:

$$
v_{i}(t) \leq t / T_{1 i}+U_{\varepsilon} \sigma_{1 i} \sqrt{t} / T_{1 i}^{3 / 2},
$$

where $U_{\varepsilon}$ is a quantile of normal distribution with the probability $1-\alpha$.

\section{Conclusions}

1. Generally, technical-economic indicators of the terminal operation should be analyzed in terms of random factors which are random with respect to any argument value as well. The arguments include time or other parameters of the terminal operation (technological process). Thus, the criteria of optimality should also be considered as being random rather than determined.

2. Most criteria used to assess the operation of the terminal and individual technological processes are interlinked and this should be taken into account when using them as optimality criteria. In determining the numerical characteristics of technicaleconomic indicators according to statistical data obtained in the process of terminal operation, mathematical expectation as well as correlation and variance functions should be calculated.

3. Random emergency situations cause failures at the transport terminal. In considering them, the theory of probability value functions should be used.

4. A great number of various factors influence the operation of the terminal and may cause its malfunction, however, their influence may differ to a great extent. Therefore, the simulation data should be optimized to be used in further decision-making.

\section{References}

1. HICKMAN, M. D. An analytic stochastic model for the transit vehicle. Transportation Science, August 2001, Vol 35, No 3, p. 215-237.

2. HAI YANG; QIANG MENG; BELL, M. G. H. Simultaneous estimation of the origin destination matrices and travel-cost coefficient for congested networks in a stochastic user equilibrium. Transportation Science, May 2001, Vol 35, No 2, p. 107-123.

3. JIAN QIAN YING.; TOSHIHIKO MIYAGL. Sensitivity analysis for stochastic user equilibrium network flows - a dual approach. Transportation Science, May 2001, Vol 35, No 2, p.124-133.

4. BAUBLYS, A. Statistical models for vehicle fleet management. Transport, 1995, 2(11), p. 5-29. 